\title{
Von Rio 1992 zu „Rio+20“. Arbeit im Kontext der aktuellen Nachhaltigkeitsdiskussion
}

Unter dem Titel „Rio+20“ fand im Juni 2012 zum zweiten Mal in Rio de Janeiro eine UNKonferenz statt, die sich mit nachhaltiger Entwicklung befasste. ${ }^{\bullet}$ Ganz im Sinne eines Jubiläums ging es primär um das erneuerte Bekenntnis zu einer nachhaltigen Entwicklung und deren Weiterentwicklung als Green Economy. Green Economy setzt auf Energie- und Ressourceneffizienz, nicht zuletzt als Motor für Wachstum und neue Arbeitsplätze. Damit gewinnt die lange Zeit marginalisierte Diskussion um die Verknüpfung von Arbeit und Nachhaltigkeit an Aktualität. Der Beitrag zeichnet markante Eckpunkte der mehrjährigen Auseinandersetzungen um diese Zusammenhänge nach und beleuchtet kritisch den Stand der Debatten.

BEATE LITTIG

\section{Nachhaltige Arbeit versus Green Jobs}

In der gegenwärtigen Nachhaltigkeitsdiskussion lassen sich zwei unterschiedliche Strategien hinsichtlich der Zukunft der Arbeit und nachhaltiger Entwicklung ausmachen. Die eine setzt auf die sozial abgesicherte Umverteilung von (Erwerbs-)Arbeit durch Arbeitszeitverkürzung bei gleichzeitiger Erweiterung des Arbeitsbegriffs und der Aufwertung nicht bezahlter, aber gesellschaftlich notwendiger Arbeitsleistungen (DIW et al. 2000; HBS 2000). ${ }^{2}$ Dieser Entwurf der sogenannten Mischarbeit hat vor allem im Kontext der wissenschaftlichen Nachhaltigkeitsforschung inzwischen einige Beachtung gefunden. Doch die politische und öffentliche Resonanz für die Überlegungen zur nachhaltigen Transformation der gegenwärtigen (Erwerbs-)Arbeitsgesellschaften in Richtung nachhaltiger Tätigkeitsgesellschaften ist eher gering.

Die andere Strategie propagiert die Schaffung von sogenannten Green Jobs, neuen sozial- und umweltverträglichen Arbeitsplätzen bzw. der entsprechenden Konversion bestehender Arbeitsplätze. ${ }^{3}$ Green Jobs sind für unterschiedliche politische Akteurinnen und Akteure lukrativ, angefangen von den Nachhaltigkeitsstrategen der Vereinten Nationen (UNO), einigen umweltpolitischen Nichtregierungsorganisationen (NGO), über die Gewerkschaften bis hin zu den nationalen Umweltministerien. Dieser Ansatz hat bereits eine längere Geschichte, auch wenn in der aktuellen, inter- nationalen Diskussion „Green Jobs“ in einer globalen „Green Economy“ auf allen politischen Ebenen mit einem gewissen Neuheitswert beworben werden. Jedenfalls kommt der Schaffung von „grünen Arbeitsplätzen“ im Rahmen einer „grünen Ökonomie“ derzeit einige mediale Aufmerksamkeit zu. ${ }^{\oplus}$

So positiv es zu bewerten ist, dass das wichtige Thema nachhaltige Entwicklung und Arbeit allmählich ins Blickfeld relevanter Akteure gerät, so werfen die skizzierten Ansätze aber auch eine Reihe von Fragen auf. Diese betreffen sowohl die zugrunde liegenden Problemdefinitionen als auch

\footnotetext{
- Rio+20. United Nations Conference on Sustainable Development, http://www.uncsd2012.org/rio20/ (letzter Zugriff: 17.07.2012)

(2) Eine umfangreiche Bestandsaufnahme und Auseinandersetzung mit Konzeptionen eines erweiterten Arbeitsbegriffs haben Littig und Spitzer (2011) vorgelegt.

3) Vgl. als Überblick Leitner et al. (2012).

(4) Vgl. etwa die Seiten des deutschen und österreichischen Umweltministeriums, http://www.bmu.de/pressemitteilungen/aktuelle_pressemitteilungen/pm/48744.php (letzter Zugriff: 17.07.2012); http://www.lebensministerium.at/umwelt. html (letzter Zugriff: 17.07.2012) oder des europäischen Parlaments, http://www.europarl.europa.eu/sides/getDoc. do ?language $=$ de\&type $=I M-P R E S S \&$ reference $=20100907 \mid$ PR81592 (letzter Zugriff: 17.07.2012.
} 
die Umsetzungshemmnisse und Realisierungschancen. Die folgenden Ausführungen stellen die beiden skizzierten Ansätze zunächst in ihren Diskussionszusammenhängen dar und diskutieren sie anschließend hinsichtlich ihrer konzeptionellen Stärken und Schwächen und möglichen politischen Wirksamkeit.

\section{Arbeit neu gedacht. Die Grundlagen nachhaltiger Arbeit}

Erst seit etwa 15 Jahren lässt sich im Diskurs über eine ökologisch, ökonomisch und sozial nachhaltige Entwicklung ein Diskussionsstrang ausmachen, der die Konsequenzen und Voraussetzungen einer nachhaltigen Neuorientierung für die gegenwärtigen (Erwerbs-)Arbeitsgesellschaften thematisiert. Die Debatte mutet zunächst akademisch an, wird sie doch in erster Linie von engagierten Wissenschaftlerinnen und Wissenschaftlern und wissenschaftlichen Einrichtungen geführt, die im Auftrag unterschiedlicher Interessengruppen Studien über die Verknüpfung von Nachhaltigkeit und Arbeit ausarbeiten. Zu nennen sind hier insbesondere zwei Studien zur zukunftsfähigen Gestaltung Deutschlands von 1996 (BUND/Misereor 1996) und 2008 (BUND et al. 2008), ${ }^{\circ}$ die beide unter anderem für eine Neudefinition und Umverteilung von Arbeit plädieren.

Das Projekt „Arbeit und Ökologie“ (DIW et al. 2000; HBS 2000) ${ }^{\odot}$ und die sogenannte Mischarbeit als zentrales Konzept von sozialer Nachhaltigkeit dient als Referenzprojekt, wenn es um die Ausbuchstabierung eines alternativen, nachhaltigen Arbeitsbegriffs geht: „Mischarbeit ist gekennzeichnet durch die Kombination verschiedener Tätigkeiten mit unterschiedlichen Gestaltungsprinzipien und Anforderungen, aus denen sich Mischqualifikationen und Mischbelastungen ergeben. Schließlich entspricht der Mischung der Arbeiten eine Kombination verschiedener Einkommen (Mischeinkommen) und das heißt eine soziale Absicherung, die nicht allein auf Erwerbsarbeit beruht, sondern aus mehreren Quellen resultiert und deren Basis eine gesellschaftliche Grundsicherung sein könnte." (Hildebrandt 2003, S. 390).

Mit diesem erweiterten Arbeitsbegriff kommt das Konzept nicht nur langjährigen Forderungen der feministischen Frauen- und Geschlechterforschung nach. ${ }^{\circ}$ Es integriert auch zentrale industrie- und arbeitssoziologische Befunde zur Krise des Fordismus (vgl. im Überblick Littig/Spitzer 2011). Insofern dient der der „Mischarbeit“ zugrunde liegende konzeptionelle Ansatz dazu, gegenwärtige Trends der gesellschaftlichen Arbeitsverhältnisse - vor allem zunehmende Flexibilisierung und Subjektivierung von Arbeit und die weitgehende Erosion des sogenannten (männlichen) Normalarbeitsverhältnisses - zu analysieren. Ausgehend von dieser Bestandsaufnahme und Bezug nehmend auf das Nachhaltigkeitsparadigma der mehrdimensionalen Vertei- lungsgerechtigkeit wurden in dem Projekt normative Leitlinien von sozialer Nachhaltigkeit bestimmt. ${ }^{\circledR}$ Dazu zählen die selbstbestimmte Lebensführung durch verschiedene Arbeitsformen im Lebensverlauf (Mischarbeit), bürgerschaftliche Partizipation, Chancengleichheit im Zugang zu grundlegenden gesellschaftlichen Ressourcen sowie eine ökologisch verträgliche Grundbedürfnisbefriedigung, die Möglichkeit zu persönlicher Entfaltung sowie die Erhaltung von Gesundheit (Brandl/Hildebrandt 2002).

Die Umsetzung von sozialer Nachhaltigkeit und Mischarbeit als deren Leitkonzept bedarf spezifischer politischer Maßnahmen. Dazu zählt prominent eine sozial-ökologische Steuerreform, also eine erhöhte Ressourcenbesteuerung und verringerte Besteuerung des Faktors Arbeit. Gleichzeitig soll über eine generelle (Erwerbs-)Arbeitszeitverkürzung (auf 25-30 Stunden pro Woche) eine Umverteilung von Arbeit erreicht werden. ${ }^{\ominus}$ Die Förderung verschiedener Lebensführungsmodelle erfordert eine entsprechende rechtliche $\mathrm{Ab}$ sicherung neuer Beschäftigungsformen, die freie Wahl des Arbeitsortes, Zeitsouveränität sowie die Sicherung der Übergänge zwischen den verschiedenen Arbeitsformen. Eine derartige Reorganisation verlangt auch nach neuen kollektiven Interessenvertretungen sowie einer Reihe von sozialen Innovationen (durch die Schaffung von Infrastrukturen wie Förderungen, Projekte, Experimente, Stiftungen).

(5) Beide Studien wurden vom Wuppertal Institut für Klima, Umwelt, Energie durchgeführt.

(6 Diese Studie wurde, gefördert von der Hans-Böckler-Stiftung, von 1996-2000 von einem Forschungsverbund des Wissenschaftszentrums Berlin für Sozialforschung (WZB) des Wuppertal Instituts für Klima, Umwelt, Energie (WI) und des Deutschen Instituts für Wirtschaftsforschung (DIW) Berlin durchgeführt.

(7) Aus deren Reihen werden inzwischen vergleichbare Konzepte diskutiert, wie etwa die Vier-in-einem-Perspektive von Frigga Haug (2008). Demnach sollen die durchschnittlichen 16-Stunden-Wachzeiten zu je einem Viertel auf Erwerbsarbeit, (Ver-)Sorge-Arbeit (Care), Gemeinschaftsarbeit und persönliche Weiterentwicklung aufgeteilt werden (können). Vgl. auch Biesecker 2008.

8 Daneben gibt es eine (vergleichsweise wenig rezipierte) Position, die dem Sozialen einen Eigenwert und eine Eigenlogik zuspricht und nachhaltige Veränderungen insbesondere unter der Perspektive sozialer Kohäsion betrachtet. Soziale Nachhaltigkeit bezieht sich in diesem Verständnis vor allem auf den schonenden Umgang mit der menschlichen Arbeitskraft als natürliche Ressource sowie auf den Zusammenhalt sozialer Entitäten (Senghaas-Knobloch 2008).

(9 Weitergehend sind die Überlegungen von Stahmer, der eine Halbtagsgesellschaft für die Verwirklichung nachhaltiger Produktions- und Konsummuster für notwendig erachtet (Stahmer 2005). 


\section{Nachhaltige Arbeit in der Krise}

Wurden die Zusammenhänge zwischen Nachhaltigkeit und Arbeit um die Jahrtausendwende nur vereinzelt thematisiert, so hat sich das Interesse an dieser Thematik jedenfalls innerhalb der Nachhaltigkeits-Community inzwischen deutlich erhöht. Dies ist nicht zuletzt dem erhöhten Krisendruck der letzten Jahre geschuldet, der zum Hinterfragen grundlegender Paradigmen, allen voran des Wirtschaftswachstums, herausfordert. Mehrere prominente sozialwissenschaftlich orientierte Nachhaltigkeitswissenschaftlerinnen und -wissenschaftler stellen die Fortschreibung der herrschenden primären Orientierung an einer Vollerwerbsgesellschaft bei steigendem Wirtschaftswachstum aufgrund der multiplen Krise grundsätzlich zur Disposition (z. B. Jackson 2009; Seidl/Zahrnt 2010; Spangenberg 2011). Begründet wird dies vor allem damit, dass eine Entkoppelung von Wirtschaftswachstum und Ressourcen- und Energieverbrauch bislang nicht gelungen ist, dass die dominante Nutzung von fossilen Energieträgern den Klimawandel beschleunigt und allein technische Lösungen zur Behebung der ökologischen Krisenphänomene in einer globalen Perspektive unzureichend sind (Schor 2005; Fischer-Kowalski/ Haberl 2007; UNEP 2012). Hinzu kommen noch ReboundEffekte, die die relativen Einsparungen durch erhöhten Verbrauch absolut steigern (Madlener/Alcott 2011).

Als Konsequenz wird aus den genannten Gründen ein deutlich verringertes Wirtschaftswachstum, die Kürzung der Erwerbsarbeitszeit, eine Erweiterung des Arbeitsbegriffs und eine Suffizienz-Orientierung des Konsums eingefordert. Diese Einschätzung wird noch durch die Feststellung einer multiplen Krise in den Bereichen des Finanzmarktes, der Wirtschaft, der Ökologie sowie Care unterstrichen (Brand 2009). Abgeleitet wird daraus die notwendige grundlegende Transformation der gegenwärtigen nicht-nachhaltigen Arbeitsgesellschaften, weil herkömmliche Krisenrezepte versagen (müssen). Denn: „Die als Lösung propagierte Wachstumspolitik ist nicht nur ökonomisch nicht erfolgreich, sie ist auch weder umwelt- noch sozialverträglich. Mit ihrem Fokus auf Deregulierung, Privatisierung und Staatsabbau unterminiert sie die politische Handlungsfähigkeit, schwächt Arbeits- und Umweltschutz und überantwortet die Gemeingüter dem privaten Gewinnstreben. [...] Gleichzeitig wird das angeblich unzureichende Wachstum der Arbeitsproduktivität als Wachstumsbremse kritisiert. Dahinter steckt die Erfahrung der Industriegesellschaft, dass durch zunehmende Standardisierung und Automatisierung die Produktivität permanent und signifikant gesteigert werden kann." (Spangenberg 2011, S. 18).

Doch die Wachstumsfrage wird in der NachhaltigkeitsCommunity nicht einhellig beantwortet (im Überblick Hinterberger et al. 2009). Sie changiert zwischen den Empfehlungen eines erhöhten oder moderaten Wachstums (Jackson 2009; UNEP 2012) bis zum Nullwachstum und der notwendigen Transition hin zu einer Postwachstumsgesellschaft (La- touch 2006; Seidl/Zahrnt 2010; Miegel 2010; Schor 2010). Grundsätzlich hinterfragt wird von den Wachstumskritikern jedenfalls die einseitige Orientierung der Wohlstandsmessung an rein ökonomischen Kennzahlen statt an einem umfassenderen Lebensqualitätsindex (zuletzt etwa Denkwerk Zukunft 2012; vgl. auch die Beiträge in Miegel/Brand 2012). Festzuhalten bleibt bei aller Heterogenität, dass die Diskussion um die Grenzen des Wachstums international deutlich an wissenschaftlicher, öffentlicher und politischer Aufmerksamkeit gewonnen hat. ${ }^{\circledR}$ Die Akteurinnen und Akteure der gegenwärtigen wachstumskritischen Debatte repräsentieren unterschiedliche gesellschaftliche Gruppierungen, was jedoch keinesfalls als hinderlich, wenn nicht sogar als begrüßenswert erscheint: „Eine von vielen gesellschaftlichen Gruppen getragene Kritik ist notwendig, um das seit Jahrzehnten verankerte Leitbild Wirtschaftswachstum zu thematisieren, zu entzaubern und sich dann von ihm zu verabschieden. Über diesen Grundkonsens hinaus und was die Gestaltungsvorstellungen anbelangt, dürften unterschiedliche Interessenlagen und gesellschaftliche Werte offenkundig werden. Hierüber ist eine weitere gesellschaftliche Diskussion angesagt.“(Seidl/Zahrnt 2010, S. 227) Unbestritten sind seitens der Wachstumskritikerinnen und -kritiker auch die notwendige Erweiterung des erwerbsarbeitszentrierten Arbeitsbegriffs sowie die Umverteilung von Erwerbsarbeit bei gleichzeitiger Arbeitszeitreduktion und entsprechender wohlfahrtsstaatlicher Absicherung. Allerdings spielt sich dieser Diskurs in einem engen Kreis von Fachleuten ab, ohne die Millionen von Betroffenen zu erreichen. Dies liegt unter anderem daran, dass sich die nach wie vor zentrale Bedeutung von Erwerbsarbeit in allen ihren Funktionen in den erweiterten Arbeitskonzepten nur zum Teil widerspiegelt (vgl. SOFI et al. 2005; SenghaasKnobloch 1998). Während die Frage der Einkommenssicherung und des wohlfahrtsstaatlichen Arrangements von den meisten dargestellten Ansätzen relativ ausführlich behandelt wird, werden die psychosozialen Funktionen der Erwerbsarbeit und ihre Bedeutung für die bürgerschaftliche Integration weitaus weniger kommentiert. Wenn Erwerbsarbeit quasi als alleinige Quelle für diese Funktionen immer mehr versiegt und demgegenüber diese Funktionen aus der Nichterwerbsarbeit geschöpft werden sollen, ist ein grundlegender soziokultureller Wandel notwendig. Wie dieser Wandel in

\footnotetext{
(11) Davon zeugt auch die aktuelle Enquete-Kommission „Wachstum, Wohlstand, Lebensqualität“ des Deutschen Bundestags, die den "Stellenwert von Wachstum in Wirtschaft und Gesellschaft ermitteln, einen ganzheitlichen Wohlstands- und Fortschrittsindikator entwickeln und die Möglichkeiten und Grenzen der Entkopplung von Wachstum, Ressourcenverbrauch und technischem Fortschritt ausloten" soll, http://www.bundestag.de/bundestag/ ausschuesse17/gremien/enquete/wachstum/index.jsp, (letzter Zugriff 17.07.2012). Vgl. auch die 2. Internationale Konferenz zu "Wachstum im Wandel”, die im Oktober 2012 vom österreichischen Lebensministerium ausgerichtet wurde, http://www.wachstumimwandel.at/konferenz2012/ (letzter Zugriff: 17.07.2012).
} 
Gang gesetzt werden soll und wer seine Träger sein sollen, wird in den meisten Studien nicht behandelt. ${ }^{\circledR}$

Inwieweit die Konzepte eines erweiterten Arbeitsbegriffs strukturelle Veränderungen bewirken können, ist schwer abschätzbar. Der Mainstream der Wirtschaftswissenschaften und der Wirtschaftspolitik geht seit den letzten 20 Jahren einhellig in Richtung Senkung der Arbeitskosten und plädiert für eine Verlängerung der Arbeitszeit zur Ankurbelung der Konsumnachfrage (vgl. Scherhorn 2005, S. 240). Politik und Staat setzen primär auf Wirtschaftswachstum als Strategie gegen Arbeitslosigkeit. Dies wird auch in der gegenwärtigen Krise als Allheilmittel propagiert. Das Thema Arbeitszeitverkürzung erscheint als eine Art Joker, den man konjunkturabhängig aus dem Hut zaubert und bei Bedarf wieder verschwinden lässt.

\section{Arbeit in der Green Economy}

Zu denjenigen, die Wirtschaftswachstum für unverzichtbar halten, gehören die Fürsprecher einer Green Economy wie sie auf UN-Ebene seit gut fünf Jahren verhandelt wird. Im Vorfeld der diesjährigen Jubiläumskonferenz „Rio+20“ der Vereinten Nationen gab es einen längeren Diskussionsprozess über die Beseitigung der Schwachstellen der avisierten nachhaltigen Entwicklung. Denn Nachbesserungsbedarf an diesem Konzept deklarierten die Verantwortlichen des UNEP (United Nation Environmental Programme) spätestens 2008. In diesem Jahr startete unter der Schirmherrschaft der UNEP die Green Economy Initiative, die einen sogenannten Green New Deal als Lösung für die sich anbahnende globale Wirtschaftskrise und die Umweltkrise verlangte. ${ }^{\text {(1) }}$ Im Zentrum stand infolgedessen bei „Rio+20“ die sogenannte Green Economy, die das breite und allzu unbestimmte Konzept einer nachhaltigen Entwicklung präzisieren soll und insofern von ihren Befürwortern als Weiterentwicklung von nachhaltiger
Entwicklung verstanden werden will. ${ }^{\circledR}$ In der Green Economy sollen durch technische, energie- und ressourceneffiziente Innovationen Wirtschaftswachstum und neue, grüne Arbeitsplätze geschaffen und damit Armut weltweit bekämpft werden. ${ }^{-0}$ Mit der Förderung von Green Jobs werden mehrere Ziele gleichzeitig verfolgt: neben dem Erhalt und der Schonung der natürlichen Lebensgrundlagen und Ressourcen geht es auch um die Erhöhung von Lebensqualität sowie die Schaffung krisensicherer und hoch qualifizierter Arbeitsplätze, die auch das Wirtschaftswachstum vorantreiben sollen.

Als Meilenstein für die internationale Green-Job-Initiative mehrerer UN-Organisationen gilt der vom UNEP finanzierte und 2008 vom World-Watch-Institute erstellte Bericht zu „Green Jobs: Towards Decent Work in a Sustainable, Low Carbon World“ (UNEP et al. 2008). Darin werden Schätzungen für die globale Zunahme von Green Jobs in den Bereichen erneuerbare Energien, Bauen, Transport, Rohstoffindustrie, Recycling, Agrar- und Forstwirtschaft vorgenommen. Die Definition von Green Jobs ist dabei sehr weit gefasst: „We define green jobs as work in agricultural, manufacturing, research and development (R\&D), administrative, and service activities that contribute substantially to preserving or restoring environmental quality. Specifically, but not exclusively, this includes jobs that help to protect ecosystems and biodiversity; reduce energy, materials, and water consumption through high efficiency strategies; de-carbonize the economy; and minimize or altogether avoid generation of all forms of waste and pollution." (UNEP et al. 2008, S. 3; vgl. auch UNEP 2011). ${ }^{\text {(B) }}$

Das vielversprechende Beschäftigungspotenzial der Green Jobs findet auch in der aktuellen EU-Strategie „Agenda 2020“ seinen Niederschlag, indem aufgrund umwelt-, wirtschaftsund beschäftigungspolitischer Überlegungen innerhalb der EU bis 2020 drei Millionen zusätzliche Green Jobs geschaffen werden sollen. Untersucht man die (politische) Werbung für die sogenannten Green Jobs, wird man feststellen, dass sie zumeist als gute, qualitätsvolle Arbeitsplätze beworben werden. ${ }^{\circledR}$ So wird auch in der Green-Job-Studie des UNEP
(11) Das eher konservativ ausgerichtete "Denkwerk Zukunft" sieht diese Fragestellung als zentral an und bemüht sich in einem Wissenschaftler-Netzwerk, mögliche Ansatzpunkte zu finden (Miegel 2010). Auch Bergmann weist immer wieder darauf hin, dass Sinnstiftung jenseits der Erwerbsarbeit und die damit verbundene Anforderung, herauszufinden, "was man wirklich, wirklich will“ (Bergmann 1997), eine schwierige und mitunter langwierige Aufgabe ist.

(12) United Nations Environment Programme: 'Global Green New Deal' - Environmentally-Focused Investment, Historic Opportunity for 21st Century Prosperity and Job Generation, http://www.unep.org/Documents.Multilingual/Default. asp?Document $\mid D=548$ \&ArticleID=5957\& $\mid=e n$, (letzter Zugriff: 17.07.2012).

(3) Dies ist dann auch derTenor des Abschlussdokuments der Konferenz 'The future we want', das neben den Aussagen zur Green Economy vielfach die 20 Jahre alten Dokumente von Rio 1992 bestätigt, http://daccess-dds-ny.un.org/doc/ UNDOC/GEN/N12/381/64/PDF/N1238164.pdf?OpenElement (letzter Zugriff: 26.06.2012).

(44) United Nations Environment Programme: 'What is the Green Economy?', http://www.unep.org/greeneconomy/ AboutGEI/WhatisGEl/tabid/29784/Default.aspx (letzter Zugriff: 17.07.2012).

(5) Ob die Definition auch die informellen Müllarbeiterinnen und Müllarbeiter im globalen Süden einschließt, ist ungeklärt.

(16) Vgl. etwa das österreichische Lebensministerium, URL: http://www.green-jobs.at/ (letzter Zugriff: 17.07.2012) oder das deutsche Umweltministerium, http://www.bmu.de/files/ pdfs/allgemein/application/pdf/green_recovery_bf.pdf (letzter Zugriff 17.07.2012). Kritisch dazu die Europäischen Grünen, http://www.elisabeth-schroedter.de/im-fokus/greenjobs (letzter Zugriff: 17.07.2012). 
auf die von der International Labour Organization (ILO) definierten Arbeitsbedingungen für sogenannte „Decent Jobs“ verwiesen: in erster Linie eine angemessene Entlohnung, $\mathrm{Si}$ cherheit am Arbeitsplatz sowie Karriereperspektiven und Arbeitnehmerrechte (UNEP et al. 2008; ILO 2008). Doch insgesamt und von wenigen Ausnahmen abgesehen spielt bislang die Arbeitsqualität in der politischen Diskussion über Green Jobs wie auch in der darauf bezogenen Forschung kaum eine Rolle (Pickshaus 2010; Kalleberg 2009; Kuhl o. J.; Mattera et al. 2009; Kurz-Scherf 2005).

\section{Zur Kritik der Green Jobs}

Eine der wenigen, kürzlich fertiggestellten Studien, die sich mit den Arbeitsbedingungen und Beschäftigungspotenzialen von Green Jobs befasst, ist eine Länderstudie über die Situation in Österreich (Leitner et al. 2012). Der gewählte Zugang zur Analyse der Arbeitsbedingungen von Green Jobs erfolgte über Wirtschaftsbranchen, um auf der Basis von Sekundärstatistiken, verfügbaren Studien und Interviews mit Expertinnen und Experten die Arbeitsbedingungen in den relevanten Branchen zu beschreiben und miteinander zu vergleichen. Im Zentrum standen die Landwirtschaft (20\% Anteil an den Green Jobs), die Bauwirtschaft (17 \%), der Handel (11\%), Energieversorgung, Architektur, Abwasser- und Abfallentsorgung (je $6 \%$ ), Tourismus (1\%) sowie das sehr heterogene produzierende Gewerbe (20\%). Aufgrund ihrer Analysen kommen die Autorinnen und Autoren zu folgenden zentralen Ergebnissen: „Zusammenfassend bleibt festzuhalten, dass derzeit zwar die Zahl der Green Jobs zunimmt, aber unklar ist, wie hoch der Nettobeschäftigungszuwachs ist. Der Anstieg der Zahl von Green Jobs ergibt sich wohl überwiegend als Folge von Umschichtungen innerhalb der Branchen, d. h. traditionelle Beschäftigungsverhältnisse werden in Green Jobs ,umgewandelt. Die Beschreibung der Situation in den ausgewählten Branchen anhand verfügbarer sekundärstatistischer Daten zeigt, dass Green Jobs deutlich heterogener sind, als sie in der öffentlichen Diskussion wahrgenommen werden. Das Bild in der Diskussion (siehe z. B. die Homepage des Lebensministeriums) ${ }^{\circledR}$ ist stark geprägt durch das Bild technischer Fachkräfte mit (zusätzlichen) Umweltqualifikationen, deren Arbeitsbedingungen nicht als prekär eingeschätzt werden. Diesem Bild entspricht am ehesten die Branche der Energieversorgung, die mit einem Anteil von $6 \%$ aller Green Jobs quantitativ jedoch nur eine begrenzte Rolle spielt" (Leitner et al. 2012, S. 82). Demgegenüber nicht thematisiert wird, dass der Großteil der Green Jobs dagegen Branchen mit schlechten Arbeitsbedingungen zugerechnet wird, wie z. B. in der Land- und Forstwirtschaft, im Bauwesen und im Handel. ${ }^{\text {() }}$

Diese Befunde sind nicht überraschend. Bereits in den 1990er Jahren gab es eine vergleichbare Diskussion über die beschäftigungsrelevanten Fragen von Umweltschutzmaßnahmen, insbesondere um mögliche Beschäftigungszuwächse (OECD 1995; Peemüller 1999). Auch damals schon wurden einige Studien erstellt, die auf die vielfach schlechte Arbeitsqualität von Jobs im Umweltbereich hingewiesen haben (Hildebrandt/Oates 1997; Ritt 1998). Nicht zuletzt wurde auch die mangelnde Berücksichtigung von frauenpolitischen Interessen im Umweltschutzsegment des Arbeitsmarkts thematisiert (Littig 1998); eine Diagnose, die auch für die aktuelle Diskussion über die Green Jobs zutrifft (Littig 2013).

Insofern bietet die internationale Green-Job-Initiative substanziell nicht viel Neues. Sie kann vielmehr als Neuauflage einer bereits länger dauernden Auseinandersetzung um Umwelt und Arbeit interpretiert werden, die offensichtlich immer wieder neue Konjunkturen erlebt; anscheinend immer dann, wenn akute Wirtschaftskrisen zu bewältigen sind. Betrachtet man die Bestrebungen für die Green Economy und die Green Jobs aus einer globalen Perspektive, sind umso mehr grundlegende Bedenken gegen diese Krisenbearbeitungsprojekte anzumelden. Unter anderem deshalb, weil die Produktion von regenerativer Energie, wie sie von der grünen Ökonomie propagiert wird, vielfach mit Landenteignungen und Vertreibung einhergeht und so insgesamt soziale Ungerechtigkeit verschärft (Heinrich-BöllStiftung 2012). Hierfür gibt es vielfältige Beispiele, etwa die Nutzung von Wasserkraft und den Bau von Staudämmen in Brasilien und China oder die Kultivierung von Palmölplantagen für Biotreibstoffe in Indonesien (Brand 2012; Brunnengräber/Haas 2012). Der Megastaudammbau und der monokulturelle Anbau von nachwachsenden Rohstoffen sind auch ökologisch hoch problematisch (Butterbach-Bahl et al. 2010). Aufgrund der geschlechterdifferenzierenden Arbeitsteilung ist davon auszugehen, dass (vor allem ärmere, auf dem Land lebende) Frauen im globalen Süden von der Ansiedelung des Agrobusiness und der Zerstörung kleinbäuerlicher Strukturen in besonderem Maße betroffen sein werden: sei es, weil sie für die Versorgung mit Wasser und Brennholz und für die Bewirtschaftung der Böden zuständig sind oder mit landwirtschaftlichem

(17) Statt der Arbeitsbedingungen im umfassenden Sinn werden in mehreren Studien speziell die Qualifikationsanforderungen von Green Jobs behandelt (OECD et al. 2010; CEDEFOP 2009).

(18) Lebensministerium, http://www.lebensministerium.at/umwelt/green-jobs.html, (letzter Zugriff: 17.07.2012).

(19) Zu einer vergleichbaren Einschätzung, nämlich einem ambivalenten Bild von Green Jobs, kommt auch eine jüngere Untersuchung für die USA (Mattera et al. 2009). Anhand dieser wurden sowohl Bereiche identifiziert, in denen Niedrigeinkommen dominieren und keine Arbeitnehmervertretungen aktiv waren, als auch Bereiche, in denen die erwarteten "good, middle-class jobs" anzutreffen waren. Die Autoren stellen daher fest: „Green jobs are not automatically good jobs. We have to make them so." (ebd., S. 6). 
Handel Einkommen generieren müssen (vgl. Appel 2010). Insgesamt ist zu vermuten, dass die Green Economy die sozialen und ökologischen Widersprüche des Kapitalismus nicht wird lösen können (Altvater 2011), sondern die Probleme lediglich in die Zukunft verlagert: „Letztlich wird es sich um ein exklusives, auf den globalen Norden und die aufstrebenden Teile des globalen Südens beschränktes Modernisierungsprojekt handeln, das neue innergesellschaftliche und internationale Ausschließungen hervorbringt und durch imperialistische Beziehungen zu anderen Teilen der Welt abgesichert wird." (Wissen 2012, S. 34).

Den skeptischen Ergebnissen der zitierten Studien ist noch hinzuzufügen, dass die Wachstumspotenziale der Green Economy und der Green Jobs in erheblichem Maß von einer förderlichen Gesetzgebung und entsprechenden öffentlichen Subventionen abhängen. Insbesondere Letztere sind in Zeiten eng geschnürter Sparpakete alles andere als sicher. ${ }^{20}$

Im Kontext einer umfassenden Nachhaltigkeitsdiskussion, aber auch für eine möglichst rasche Reduktion der für den Klimawandel verantwortlichen Emissionen sowie biodiversitätsförderlichen Maßnahmen sind die sogenannten Green Jobs bestenfalls ein kleiner Baustein in einer notwendigen größeren sozial-ökologischen Transition (FischerKowalski/Haberl 2007). Auch wenn sie das Potenzial haben, in einem begrenzten Ausmaß neue und qualitätsvolle Arbeitsplätze zu schaffen, insbesondere in den wissensintensiven Branchen, so sind sie jedoch weit davon entfernt, die multiple Krise lösen zu können.

\section{Resümee und Ausblick}

Im vorliegenden Beitrag wurden zwei zentrale Diskussionsbeiträge zum Thema Arbeit und nachhaltige Entwicklung unterschieden. Der eine lässt sich kurz als alternatives, nachhaltiges Arbeitsverständnis bezeichnen, der andere als Green-Job-Initiative. Beide Ansätze wurden als unterschiedliche Krisenbearbeitungsstrategien charakterisiert. Es wurde insbesondere die mangelnde öffentliche Resonanz im einen Fall und die politische Kurzsichtigkeit im andern Fall hervorgehoben. Dennoch soll beiden Strategien nicht grundsätzlich soziale Innovationskraft abgesprochen werden. Es scheint derzeit noch nicht ausgemacht zu sein, welche Verwirklichungschancen die grüne Ökonomie hat. So begrüßenswert das Versprechen eines grünen Wirtschaftens auch sein mag, ist Skepsis anzumelden, nicht nur aus geschlechterpolitischer Sicht, sondern auch im Hinblick auf die Widersprüchlichkeit des Konzepts (Brand 2012; Wichterich 2012). Die Realisierungschancen eines erweiterten Arbeitsverständnisses und die konsequente soziale Umverteilung von Arbeit sind ebenfalls schwer abzuschätzen. Hier mangelt es nicht zuletzt an politischen Akteurinnen und Akteuren wie auch Allianzen, die ein solches Transforma- tionsprojekt vorantreiben könnten. Entgegen einem eklektischen Umgang mit dem Thema Umverteilung von Arbeit plädieren einige NGOs für ein „politisches Projekt, in dem Gewerkschaften, Sozialprotest-Initiativen, linke Strömungen in den Parteien, Kirchen und Attac motiviert werden", zusammen für ein Projekt von Arbeitszeitverkürzung, Mindestlohn und Grundeinkommen zu kämpfen (Krull et al. 2009). Erweitert werden könne die Allianz von der Frauenbewegung (ebd. S. 9) und aufgeschlossenen Teilen der Mittelschicht (ebd. S. 24). Attac etwa setzt sich für einen $\mathrm{Zu}$ sammenschluss der Zivilgesellschaft mit einzelnen Sympathisanten in den Parteien ein, um so den Druck zu erhöhen und erweiterte Arbeitskonzepte umsetzen zu können. Diese Forderung liest sich recht unbestimmt; dementsprechend ist diese Allianz auch (noch) nicht in Sicht und scheint weder bei Attac noch bei den Gewerkschaften oder Kirche Priorität zu haben.

Aber auch wenn die Ansätze viele Fragen offen lassen und unterschiedliche Zielsetzungen verfolgen, so müssen sich beide Strategien mit Blick auf die Gestaltung einer nachhaltigen Arbeitsgesellschaft nicht gegenseitig ausschließen, vielmehr können sie sich sinnvoll ergänzen. Ähnlich argumentiert auch Holzinger (2010) in einer Auftragsstudie des österreichischen Lebensministeriums zur Zukunft der Arbeit, in der er drei Szenarien entwirft. Potenzial für die Schaffung neuer Arbeitsplätze (Szenario 1) besteht nach seiner Einschätzung (ebd. S. 78) in erster Linie im Bereich der industrienahen und vor allem der personennahen Dienstleistungen. Die Umverteilung von Arbeit (Szenario 2) ließe sich durch Arbeitszeitverkürzung mit oder ohne Lohnausgleich erreichen, zum Teil auch durch flexiblere Arbeitszeitlösungen (Lebensarbeitszeit, Auszeiten, Teilzeit etc.). Die Finanzierung hängt letztendlich von den konkreten Modellen ab und wäre sozialpartnerschaftlich auszuhandeln. Das dritte Szenario „Anders arbeiten“ meint bei Holzinger - vergleichbar dem Konzept der Mischarbeit eine Aufwertung gesellschaftlich notwendiger Arbeiten jenseits der Erwerbsarbeit, also der Versorgungs-, Eigenund Gemeinschaftsarbeit sowie eine geschlechtergerechte Aufteilung dieser Arbeiten. Der Wandel hin zu einer geschlechtergerechten Tätigkeitsgesellschaft bedarf staatlicher Transferleistungen oder fiskalischer Steuerung und basiert auf einem gemischten Einkommen, das sich aus verschiedenen Quellen speist. Auch in dieser Hinsicht gibt es Parallelen zum Konzept der Mischarbeit, das bei Holzinger "plurale Arbeit" heißt (ebd. S. 81).

Hinzuzufügen ist den genannten Szenarien noch ein weiteres, das mit „alternative Lebens- und Arbeitsformen“

(20) Die Streichung von Subventionen hat in Deutschland in diesem Jahr zu mehreren Insolvenzen in der Solar- und Windkraftindustrie geführt, vgl. http://www.wiwo.de/unternehmen/mittelstand/windkraft-und-solarenergie-zukunftstechnik-wird-zur-pleitebranche/6344790.html (letzter Zugriff: 17.07.2012). 
zu überschreiben ist. Gemeint sind damit verschiedene Formen genossenschaftlichen Wirtschaftens und alternative Formen des Zusammenlebens und Zusammenarbeitens, wie sie etwa in Projekten der solidarischen Ökonomie (Altvater/Sekler 2006), des vorsorgenden Wirtschaftens (Hofmeister 1999; Biesecker 2011) oder der Subsistenzwirtschaft (BennholdtThomsen 2006; Bennholdt-Thomsen/Mies 1997) praktiziert werden. ${ }^{2}$ Auch wenn diese Konzepte und Projekte aus dem Umfeld der alternativ- und globalisierungskritischen und ökofeministischen Bewegungen unrealistisch oder gar utopisch erscheinen mögen, so können sie gerade in Krisenzeiten zu Lieferanten innovativer Ideen und reflektierter Erfahrungen aus sozialen Experimenten werden. Inwieweit sie sozial verallgemeinerbar sind, bleibt jedoch eine offene Frage.

Den einzig gangbaren Weg zur Lösung der multiplen Krise wird es nicht geben, schon gar nicht global betrachtet. Insofern ist es notwendig, in unterschiedlichen Szenarien zu denken.

(29) 2012 hat die UN zum Jahr der Kooperativen deklariert, um auf die weltweite hohe Verbreitung dieser Wirtschaftsform hinzuweisen, http://social.un.org/ coopsyear/ (letzter Zugriff: 17.07.2012).

\section{LITERATUR}

Altvater, E. (2011): Mit Green New Deal aus dem Wachstumsdilemma?, in: WIDERSPRUCH - Beiträge zu sozialistischer Politik 31 (60), S. 119-132, http:// www.widerspruch.ch/sites/widerspruch.ch/files/pdf/wsp60_altvater.pdf, (letzter Zugriff: 17.07.2012)

Altvater, E./Sekler N. (2006) (Hrsg.): Solidarische Ökonomie, Reader des Wissenschaftlichen Beirats von Attac, Hamburg

Appel, A. (2010): Die Genderbilanz des Klimadiskurses. Von der Schieflage einer Debatte, in: Kurswechsel (2), S. 52-62

Bennholdt-Thomsen, V. (2006): Subsistenzwirtschaft, Globalwirtschaft, Regionalwirtschaft, in: Jochimsen, M. A./Knobloch, U. (Hrsg.): Lebensweltökonomie in Zeiten wirtschaftlicher Globalisierung, Bielefeld, S. 65-88

Bennholdt-Thomsen, V./Mies, M. (1997): Eine Kuh für Hillary. Die Subsistenz perspektive, München

Bergmann, F. (1997): Die neue Arbeit. Skizze mit Vorschlag, in: Gewerkschaftliche Monatshefte 48 (9/10), S. 524-534

Biesecker, A. (2008): Kürzer Arbeiten - besser für die Umwelt. Arbeitszeitverkürzung und Ökologie, in: Zimpelmann, B./Endl, H.-L.: Zeit ist Geld. Ökonomische, ökologische und soziale Grundlagen von Arbeitszeitverkürzung, Hamburg, S. 55-76

Biesecker, A. (2011): Vorsorgendes Wirtschaften, in: Rätz, W./Egan-Krieger, T./ Muraca, B./Passadakis, A./Schmelzer, M./Vetter, A. (Hrsg.): Ausgewachsen!, Hamburg, S. 75-85

Brand, U. (2009): Die multiple Krise. Dynamik und Zusammenhang der Krisendimensionen, Anforderungen an politische Institutionen und Anforderungen an progressive Politik, hrsg. von der Heinrich-Böll-Stiftung, http://www.boell. de/downloads/internationalepolitik/multiple_krisen_u_brand_1.pdf (letzter Zugriff: 17.07.2012)

Brand, U. (2012): Green Economy - the Next Oxymoron? No Lessons Learned from Failures of Implementing Sustainable Development, in: GAIA 21 (1), S. $28-32$

Brandl, S./Hildebrandt, E. (2002): Zukunft der Arbeit und soziale Nachhaltigkeit, Opladen

Brunnengräber, A./Haas, T. (2012): Rio+20: Die grüne Beliebigkeit, in: Blätter für deutsche und internationale Politik (2), S. 15-18, http://www.blaetter.de/ archiv/jahrgaenge/2012/februar/rio20-die-gruene-beliebigkeit (letzter Zugriff: 17.07.2012
Bund für Umwelt- und Naturschutz Deutschland (BUND)/Evangelischer Entwicklungsdienst „Brot für die Welt“ (EED) (Hrsg.) (2008): Zukunftsfähiges Deutschland in einer globalisierten Welt. Ein Anstoß zur gesellschaftlichen Debatte, Frankfurt a. M.

BUND/Misereor (Hrsg.) (1996): Zukunftsfähiges Deutschland. Ein Beitrag zu einer global nachhaltigen Entwicklung, Studie des Wuppertal-Instituts für Klima, Umwelt, Energie, Basel/Boston/Berlin

Butterbach-Bahl, K./Leible, L./Kälber, S./Kappler, G./Kiese, R. (2010):Treibhausgasbilanz nachwachsender Rohstoffe - eine wissenschaftliche Kurzdarstellung, Karlsruhe Institute of Technology, KIT Scientific Reports (7556), http:// www.itas.fzk.de/deu/lit/2010/buua10a.pdf (letzter Zugriff: 17.07.2012)

Denkwerk Zukunft. Stiftung kulturelle Erneuerung (Hrsg.) (2012): Das Wohlstandsquintett - Zur Messung des Wohlstands in Deutschland und anderen früh industrialisierten Ländern. Memorandum, http://www.denkwerkzukunft. de/index.php/aktivitaeten/index/Wohlstandsquintett (letzter Zugriff:

17.07.2012)

Deutsches Institut für Wirtschaftsforschung (DIW)/Wuppertal Institut für Klima, Umwelt,Energie (WI)/Wissenschaftszentrum Berlin für Sozialforschung (WZB) (2000): Arbeit und Ökologie, Projektabschlussbericht, Hans-Böckler-Stiftung, Düsseldorf

European Centre for the Development of Vocational Training (CEDEFOP) (2009): Future skill needs for the green economy, Publications Office of the European Union, Luxembourg, http://www.cedefop.europa.eu/EN/Files/5501 en.pdf (letzter Zugriff: 17.07.2012)

Fischer-Kowalski, M./Haberl, H. (2007) (Hrsg.): Socioecological Transitions and Global Change, Cheltenham

Fischer-Kowalski, M./Swilling, M./Von Weizsäcker, E. U./Ren, Y./Moriguchi, Y./ Crane, W./Krausmann, F./Eisenmenger, N./Giljum, S./ Hennicke, P./Romero Lankao, P./Siriban Manalang, A./Sewerin S./United Nations Environment Programme (UNEP) (Hrsg.) (2011): Decoupling natural resource use and environmental impacts from economic growth, A Report of the Working Group on Decoupling to the International Resource Panel, http://www.unep.org/ resourcepanel/decoupling/files/pdf/decoupling_report_english.pdf (letzter Zugriff: 17.07.2012)

Hans-Böckler-Stiftung (HBS) (Hrsg.) (2000): Wege in eine nachhaltige Zukunft. Ergebnisse aus dem Verbundprojekt Arbeit und Ökologie, Düsseldorf

Haug F. (2008): Die Vier-in-einem-Perspektive. Politik von Frauen für eine neue Linke, Hamburg

Heinrich-Böll-Stiftung (Hrsg.) (2012): Kritik der grünen Ökonomie. Impulse für eine sozial und ökologisch gerechte Zukunft, Band 22 der Schriftenreihe Ökologie, Berlin, http://www.boell.de/downloads/Kritik_der_gruenen_Oekonomie_ kommentierbar.pdf (letzter Zugriff: 17.06.2012)

Hildebrandt, E. (2003): Arbeit und Nachhaltigkeit. Wie geht das zusammen?, in: Linne, G./Schwarz, M. (Hrsg.) (2003): Handbuch nachhaltige Entwicklung, Opladen, S. 381-393

Hildebrandt, E./Oates, A. (1997): Work, Employment and Environment. Quality and Quantity of Work in the Environmental Labour Market and its Regulation, Wissenschaftszentrum Berlin für Sozialforschung, Discussion Paper FS II 97-208, Berlin

Hinterberger, F./Hutterer, H./Omann, I./Freytag, E. (Hrsg.) (2009): Welches Wachstum ist nachhaltig? Ein Argumentarium, Wien

Hofmeister, S. (1999): Über die Produktivität des Reproduktiven. Der Beitrag des Konzepts "Vorsorgen des Wirtschaftens" zum Nachhaltigkeitsdiskurs, in: Weller, I./Hoffmann, E./Hofmeister, S. (Hrsg.): Nachhaltigkeit und Feminismus: Neue Perspektiven - Alte Blockaden, Bielefeld, S. 73-98

Holzinger, H. (2010): Zur Zukunft der Arbeit, Österreichisches Lebensministerium, Zukunftsdossier (2), http://www.wachstumimwandel.at/wp-content/ uploads/zur-zukunft-der-arbeit1.pdf (letzter Zugriff: 17.07.2012) International Labour Organization (ILO) (2008): Global Challenges for Sustainable Development: Strategies for Green Jobs, ILO Background Note, G8 Labour and Employment Ministers Conference Niigata, Japan, 11 to 13 May 2008, http://www.ilo.org/public/english/bureau/dgo/speeches/somavia/2008/ g8paper.pdf (letzter Zugriff: 17.07.2012)

Jackson, T. (2009): Prosperity without growth? - The transition to a sustainable economy, http://www.sd-commission.org.uk/data/files/publications/prosperity without_growth_report.pdf (letzter Zugriff: 17.07.2012)

Kalleberg, A. L. (2009): Precarious work, insecure workers. Employment relations in transition, in: American Sociological Review 74 (1), S. 1-22

Krull, S./Massarrat, M./Steinrücke, M. (Hrsg.) (2009): Schritte aus der Krise. Arbeitszeitverkürzung, Mindestlohn, Grundeinkommen: Drei Projekte, die zusammengehören, Reader der Attac AG ArbeitFairTeilen, Hamburg 
Kuhl, M. (о. J.):The Gender Dimensions of the Green New Deal. Study commissioned by The Greens/EFA Group in the European Parliament, http://www elisabeth-schroedter.de/im-fokus/green-jobs/green-jobs/GenderAspectsGNDfinal.pdf (letzter Zugriff: 17.07.2012)

Kurz-Scherf, I. (2005): Qualitätskriterien von Arbeit - ein Überblick, in: WSIMitteilungen 58 (4 ), S. 193-199, http://www.boeckler.de/wsimit_2005_04_kurz scherf.pdf

Latouche, S. (2006): Le pari de la décroissance, Paris (engl. Farewell to Growth, New York)

Leitner, A./Wroblewski, A./Littig, B. (2012): Green Jobs. Diskussion von Arbeitsbedingungen und Beschäftigungspotentialen, Projektbericht des Instituts für Höhere Studien, hrsg. von der Arbeiterkammer Wien, Informationen zur Umweltpolitik (186)

Littig, B. (1998): Umwelt und Arbeit - und Frauen? Über die Defizite einer Debatte, in: Ritt, T. (Hrsg.): Umwelt und Arbeit - Bestandsaufnahmen und Perspektiven, Arbeiterkammer Wien, Informationen zur Umweltpolitik (124),

S. $145-158$

Littig, B. (2013): Green Economy, Green Jobs - und Frauen? Geschlechterpolitische Überlegungen zum aktuellen Nachhaltigkeitsdiskurs, in: Appelt, E./ Aulenbacher, B./Wetterer, A. (Hrsg.): Gesellschaft - Feministische Krisendiagnosen, Münster (im Erscheinen)

Littig, B./Spitzer, M. (2011): Arbeit neu. Erweiterte Arbeitskonzepte im Vergleich, Hans-Böckler-Stiftung, Arbeitspapier (229), Düsseldorf

Madlener, R./Alcott, B. (2011): Herausforderungen für eine technisch-ökonomische Entkoppelung von Naturverbrauch und Wirtschaftswachstum unter besonderer Berücksichtigung der Systematisierung von Rebound-Effekten und Problemverschiebungen, Bericht an die Enquete-Kommission „Wachstum, Wohlstand, Lebensqualität" des Deutschen Bundestages, Kommissionsmaterialien (M-17[26]13), 12. Dez., http://www.bundestag.de /bundestag/ ausschuesse17/gremien/enquete/wachstum/gutachten/m17-26-13.pdf (letzter Zugriff: 17.07.2012)

Mattera, P./Dubro, A./Gradel, T./Tompson, R./Gordon, K./Foshay E. (2009): High road or low road? Job quality in the new green economy. Good Jobs First, Washington, http://www.goodjobsfirst.org/sites/default/files/docs/pdf/ gjfgreen jobsrpt.pdf (letzter Zugriff: 17.02.2012)

Miegel, M. (2010): Exit. Wohlstand ohne Wachstum, Berlin

Miegel, M./Brand, U. (2012) (Hrsg): Wohlstand ohne Wachstum?, in: Aus Politik und Zeitgeschichte. Beilage zur Wochenzeitung Das Parlament 62 (27-28),

S. $3-14$

Organisation for Economic Co-operation and Development (OECD) (1995):

Environmental Policies and Environment, Paris

Organisation for Economic Co-operation and Development (OECD)/MartinezFernandez, C./Hinojosa, C./Miranda, G. (2010): Green jobs and skills: the local labour market implications of addressing climate change, working document CFE/LEED, OECD, 8 February, http://www.oecd.org/dataoecd /54/43/446,83169 pdf (letzter Zugriff: 17.07.2012)

Peemüller, G. (1999): Beschäftigung im und durch Umweltschutz - Ein Literaturbericht, in: Mitteilungen aus der Arbeitsmarkt- und Berufsforschung 32 (3), S. $331-350$

Pickshaus, K. (2010): Krise und Restrukturierung: Anforderungen an eine GuteArbeit-Strategie, in: WSI-Mitteilungen 63 (7), S. 385-387, http://www.boeckler. $\mathrm{de} / \mathrm{cps} / \mathrm{rde} / \mathrm{xbcr} / \mathrm{hbs} /$ wsimit 201007 pickshaus.pdf

Ritt, T. (1998): Umwelt und Arbeit - Arbeitsbedingungen im Umweltsektor in Österreich und Deutschland. Die ignorierte Qualität der Umweltarbeit, in: WSI Mitteilungen 51 (8), S. 552-562

Scherhorn, G. (2005): Zum Widerstand gegen Arbeitszeitverkürzung, in: GAIA 14 (3), S. $240-242$
Schor, J. (2005): Sustainable Consumption and Worktime Reduction, in: Journal of Industrial Ecology 9 (1-2), S. 37-50

Schor, J. (2010): Plenitude. The New Economics of True Wealth, Berkeley

Seidl, I./Zahrnt A. (2010) (Hrsg.): Postwachstumsgesellschaft. Konzepte für die Zukunft, Marburg

Seidl, I./Zahrnt A. (2010): Verbindungslinien: Inhaltliche Zusammenhänge zwischen den Themen, in: Seidl, I./Zahrnt A. a.a.O., S. 221-228

Senghaas-Knobloch, E. (1998): Von der Arbeits- zurTätigkeitsgesellschaft? Politikoptionen und Kriterien zur ihrer Abschätzung, in: Feministische Studien 2 (98), S. $9-30$

Senghaas-Knobloch, E. (2008): Flexible Arbeitsformen als Herausforderung der sozialen Nachhaltigkeit, in: Becke, G. (Hrsg.): Soziale Nachhaltigkeit in flexiblen Arbeitsstrukturen. Problemfelder und arbeitspolitische Gestaltungsperspektiven, Münster, S. 27-43

Soziologisches Forschungsinstitut (SOFI)/Institut für Arbeitsmarkt- und Berufsforschung (IAB)/Institut für Sozialwissenschaftliche Forschung (ISF)/Internationales Institut für Empirische Sozialökonomie (INIFES) (Hrsg.) (2005): Berichterstattung zur sozioökonomischen Entwicklung in Deutschland - Arbeitsund Lebensweisen. Erster Bericht, Wiesbaden

Senatsverwaltung für Arbeit, Berufliche Bildung und Frauen (Hrsg.) (1998): Die Sackgassen der Zukunftskommission. Streitschrift wider die Kommission für Zukunftsfragen der Freistaaten Bayern und Sachsen, Schriftenreihe der Senatsverwaltung für Arbeit, Berufliche Bildung und Frauen, Bd. 33, Berlin Spangenberg, J. H. (2011): Die Grenzen der Natur setzen neue Signale. Arbeitsgesellschaft im Wandel, in: Oekom e.V. - Verein für ökologische Kommunikation (Hrsg.): Anders arbeiten, München, S. 15-24

Stahmer, C. (2005): Die Halbtagsgesellschaft - ein Konzept für nachhaltigere Produktions- und Konsummuster, in: GAIA 14 (3), S. 225-239

United Nations Environment Programme (UNEP) (2011): Green jobs.

Towards decent work in a sustainable, low-carbon world, http://www.unep. org/PDF/UNEPGreenJobs_report08.pdf (letzter Zugriff: 17.07.2012)

United Nations Environment Programme (UNEP) (2012): Geo-5. Global Environment Outlook, published by the United Nations Environment Programme, http://www.unep.org/geo/geo5.asp (letzter Zugriff: 17.07.2012)

United Nations Environment Programme (UNEP)/International Labour Organization (ILO)/International Organisation of Employers (IOE)/International Trade Union Confederation (ITUC) (Hrsg) (2008): Green Jobs: Towards Decent Work in a Sustainable, Low Carbon World, http://www.unep. org/PDF/UNEPGreenJobs_report08.pdf (letzter Zugriff: 17.06.2012)

Wichterich, Ch. (2012): Die Zukunft, die wir wollen - Eine Feministische Perspektive, Heinrich-Böll-Stiftung, Berlin

Wissen, M. (2012): Post-neoliberale Hegemonie? Zur Rolle des Green-Economy Konzepts in der Vielfachkrise, in: Kurswechsel (2), S. 28-36

\section{AUTORIN}

BEATE LITTIG, PD Dr. Dipl.-Soz., Abteilungsleiterin der Soziologie am Institut für Höhere Studien in Wien. Arbeitsschwerpunkte: Sozialwissenschaftliche Umwelt- und Technikforschung, qualitative Forschungsmethoden, Gender Studies.

littig@ihs.ac.at 